\title{
2 AC-Driven Transport Through Molecular Wires
}

\author{
Peter Hänggi, Sigmund Kohler, Jörg Lehmann, and Michael Strass \\ Institut für Physik, Universität Augsburg, Universitätsstraße 1, D-86135 \\ Augsburg, Germany
}

\begin{abstract}
We consider electrical transport properties of a molecular wire under the influence of time-dependent electromagnetic fields. A formalism based on Floquet theory is derived which allows one to calculate both the dc current through the molecular wire and the associated noise power. Approximations for the case of a weak wire-lead coupling are studied in detail.
\end{abstract}

\subsection{Introduction}

Owing to the recent experimental progress in the fabrication and characterisation of nanostructures involving single or a few molecules, the research field of molecular electronics currently enjoys a vivid activity, as exemplified by the present volume. Thereby, the main focus has been put on contacting single molecules by nanoelectrodes. This allows one to apply a transport voltage and to measure the resulting electrical current [1-8]. For the corresponding theoretical investigations, two lines of research are presently followed. The one is the ab-initio computation of the orbitals relevant for the motion of excess charges through the molecular wire [9-12], as described in Chaps. 3-6. The other line employs rather universal models to gain a qualitative understanding of the transport mechanisms involved [13-19]. Two particular problems addressed within model calculations are the conduction mechanism in the presence of electron-phonon coupling [14] and the length dependence of the current-voltage characteristics $[13,17]$. The present work also employs rather general models: We describe the molecules by a linear arrangement of tight-binding levels with the terminating sites attached to leads.

Typical energy scales of molecules lie in the infrared regime where most of today's lasers work. Hence, lasers represent a natural possibility to excite the electrons of the molecular wire and, thus, to study the corresponding changes of the conduction properties [20]. One particular question in this context is the influence of excitations by electromagnetic fields and oscillatory gate voltages on the electron transport. Such excitations bear intriguing phenomena like photon-assisted electron tunnelling [21-23] and quantum ratchet effects $[24,25]$. From a fundamental point of view, these effects are of interest because the external fields enable selective electron excitations and allow one to study their interplay with the underlying transport mechanism. In prac-

P. Hänggi et al.: AC-Driven Transport Through Molecular Wires, Lect. Notes Phys. 680 55-75 (2005)

www.springerlink.com $\quad$ (c) Springer-Verlag Berlin Heidelberg 2005 
tical applications, time-dependent effects can be used to control and steer currents in coherent conductors. Such control schemes can be valuable, however, only if they operate at tolerable noise levels. Thus, the corresponding current noise is also of prominent interest.

An experimental starting point for the investigation of the influence of electromagnetic fields on molecular conduction is the laser excitation of electrons to higher orbitals of the contacted molecule. The resulting changes of the current through a contacted molecule due to the influence of a laser field are studied. In particular, we focus on the modification of the length dependence of the conductivity [26-28]. At present, the corresponding experiments are attempted, but still no clearcut effect has been reported. The moleculelead contacts seem stable even against relatively intense laser fields, but a main problem is the exclusion and suppression of side effects like, e.g. heating of the break junction which otherwise might distort the molecule-tip setup and, thus, be responsible for the observed enhancement of the conductance.

\subsection{Basic Concepts}

\subsubsection{Model for Driven Molecular Wire Coupled to Leads}

The entire setup of the ac-driven molecular wire coupled to leads is described by the time-dependent Hamiltonian

$$
H(t)=H_{\text {wire }}(t)+H_{\text {leads }}+H_{\text {contacts }},
$$

where the different terms correspond to the molecular wire, the leads, and the wire-lead couplings, respectively. We focus on the regime of coherent quantum transport where the main physics at work occurs on the wire itself. In doing so, we neglect other possible influences originating from driving induced hot electrons in the leads, dissipation on the wire and, as well, electron-electron interaction effects. Then, in a tight-binding (Hückel) approximation with $N$ localised atomic orbitals $|n\rangle$, the wire Hamiltonian reads

$$
H_{\text {wire }}(t)=\sum_{n, n^{\prime}} H_{n n^{\prime}}(t) c_{n}^{\dagger} c_{n^{\prime}}
$$

The fermion operators $c_{n}, c_{n}^{\dagger}$ annihilate and create, respectively, an electron in the orbital $|n\rangle$. The influence of an applied ac field with frequency $\Omega=2 \pi / \mathcal{T}$ results in a periodic time-dependence of the wire Hamiltonian: $H_{n n^{\prime}}(t+\mathcal{T})=$ $H_{n n^{\prime}}(t)$. The leads are modelled by ideal electron gases,

$$
H_{\text {leads }}=\sum_{q}\left(\epsilon_{\mathrm{L} q} c_{\mathrm{L} q}^{\dagger} c_{\mathrm{L} q}+\epsilon_{\mathrm{R} q} c_{\mathrm{R} q}^{\dagger} c_{\mathrm{R} q}\right)
$$

where $c_{\mathrm{L} q}^{\dagger}\left(c_{\mathrm{R} q}^{\dagger}\right)$ creates an electron in the state $|\mathrm{L} q\rangle(|\mathrm{R} q\rangle)$ with energy $\epsilon_{\mathrm{L} q}$ $\left(\epsilon_{\mathrm{R} q}\right)$ in the left (right) lead. The tunnelling Hamiltonian 


$$
H_{\text {contacts }}=\sum_{q}\left(V_{\mathrm{L} q} c_{\mathrm{L} q}^{\dagger} c_{1}+V_{\mathrm{R} q} c_{\mathrm{R} q}^{\dagger} c_{N}\right)+\text { h.c. }
$$

establishes the contact between the sites $|1\rangle,|N\rangle$ and the respective lead, as sketched in Fig. 2.1. This tunnelling coupling is described by the spectral density

$$
\Gamma_{\ell}(\epsilon)=2 \pi \sum_{q}\left|V_{\ell q}\right|^{2} \delta\left(\epsilon-\epsilon_{\ell q}\right)
$$

of lead $\ell$, with $\ell=\mathrm{L}, \mathrm{R}$. Assuming that the lead modes are dense, $\Gamma_{\ell}(\epsilon)$ becomes a smooth function. Although we will derive expressions for arbitrary spectral density, we here remark that, often, one is interested mainly in the properties of the molecular wire itself and not in the details of the wirelead coupling. Then it is convenient to assume, in the relevant regime, the spectral density to be energy-independent and, thus, to employ the so-called wide-band limit $\Gamma_{\ell}(\epsilon) \rightarrow \Gamma_{\ell}=$ const.

To fully specify the dynamics, we choose as an initial condition for the left/right lead a grand-canonical electron ensemble at temperature $T$ and electro-chemical potential $\mu_{\mathrm{L} / \mathrm{R}}$, respectively. Then, at initial time $t_{0}$, the only nontrivial expectation values of the lead operators read

$$
\left\langle c_{\ell^{\prime} q^{\prime}}^{\dagger} c_{\ell q}\right\rangle=f_{\ell}\left(\epsilon_{\ell q}\right) \delta_{\ell \ell^{\prime}} \delta_{q q^{\prime}}
$$

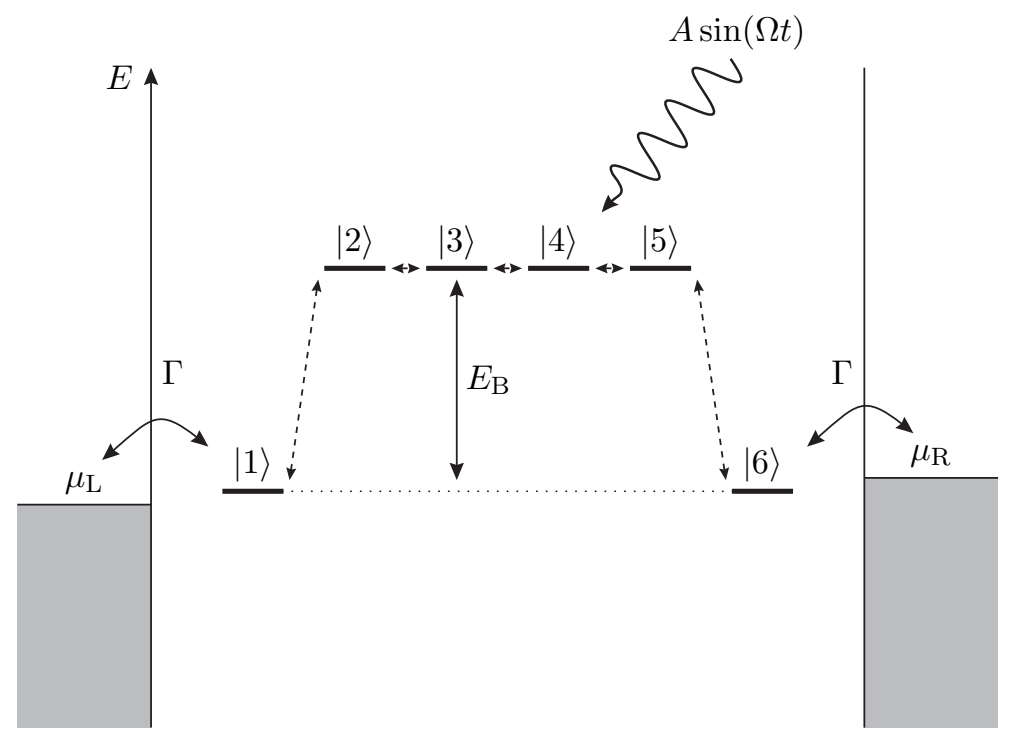

Fig. 2.1. Level structure of a molecular wire bridge consisting $N=6$ orbitals. The two end sites are coupled to two leads with chemical potentials $\mu_{\mathrm{L}}$ and $\mu_{\mathrm{R}}=\mu_{\mathrm{L}}+\mathrm{eV}$ and are bridged by the $N-2$ orbitals lying $E_{\mathrm{B}}$ above in energy. All adjacent orbitals are coupled by the tunnelling matrix elements $\Delta$. A laser field with frequency $\Omega$ and amplitude $A$ acts on the molecular wire 
where $f_{\ell}(\epsilon)=\left(1+\exp \left[\left(\epsilon-\mu_{\ell}\right) / k_{\mathrm{B}} T\right]\right)^{-1}$ denotes the Fermi function in lead $\ell$ and $k_{\mathrm{B}}$ is the Boltzmann constant. An applied voltage $V$ maps to a chemical potential difference $\mu_{\mathrm{R}}-\mu_{\mathrm{L}}=e V$ with $-e$ being the electron charge.

\subsubsection{Current Through Static Molecular Wire}

Before elucidating the influence of a driving field, let us first briefly review the scattering approach which applies to the corresponding static situation, i.e., without driving. In the absence of interactions, the current through a nanosystem like a molecular wire considered here, can be expressed in terms of the transmission $T(E)$, i.e., the probability for an electron of a given energy $E$ being transmitted from one lead to the other. This allows one to write the current as

$$
I=\frac{e}{2 \pi \hbar} \int \mathrm{d} E\left[f_{\mathrm{R}}(E)-f_{\mathrm{L}}(E)\right] T(E) .
$$

For the evaluation of the transmission, one often uses the relation [29-31]

$$
T(E)=\operatorname{tr}\left[G^{\dagger}(E) \Sigma_{\mathrm{R}}(E) G(E) \Sigma_{\mathrm{L}}(E)\right],
$$

which expresses the transmission in terms of the retarded Green function $G(E)$ of the system and the self-energies $\Sigma_{\mathrm{L} / \mathrm{R}}(E)$ resulting from the coupling to the leads. The trace in the last equation sums over all single-particle states of the wire.

Besides the mean value of the current, another quantity of interest is its fluctuation, i.e., the current noise which is described by the symmetrised correlation function

$$
S_{\ell}\left(t, t^{\prime}\right)=\frac{1}{2}\left\langle\Delta I_{\ell}(t) \Delta I_{\ell}\left(t^{\prime}\right)+\Delta I_{\ell}\left(t^{\prime}\right) \Delta I_{\ell}(t)\right\rangle
$$

of the current fluctuation operator $\Delta I_{\ell}(t)=I_{\ell}(t)-\left\langle I_{\ell}(t)\right\rangle$. For a stationary process, the correlation function $S\left(t, t^{\prime}\right)=S\left(t-t^{\prime}\right)$ is a function of only the time difference. The noise strength can be characterised by the zero-frequency component of $S_{\ell}(\tau)$,

$$
\bar{S}_{\ell}=\int_{-\infty}^{\infty} \mathrm{d} \tau S_{\ell}(\tau) .
$$

It can be shown that due to charge-conservation, for two-terminal devices $\bar{S}_{\ell}$ is independent of the contact $\ell$, i.e., $\bar{S}_{\mathrm{L}}=\bar{S}_{\mathrm{R}} \equiv \bar{S}$. The noise strength $S$ may be expressed in terms of the transmission function $T(E)$ as [32]

$$
\begin{aligned}
& S=\frac{e^{2}}{2 \pi \hbar} \int \mathrm{d} E\left\{T(E)\left[f_{\mathrm{L}}(E)\left[1-f_{\mathrm{L}}(E)\right]+f_{\mathrm{R}}(E)\left[1-f_{\mathrm{R}}(E)\right]\right]\right. \\
& \left.+T(E)[1-T(E)]\left[f_{\mathrm{R}}(E)-f_{\mathrm{L}}(E)\right]^{2}\right\} .
\end{aligned}
$$

As a dimensionless measure for the relative noise strength, we employ the so-called Fano factor [33] 


$$
F=\frac{\bar{S}}{e|\bar{I}|}
$$

where $\bar{I}$ denotes the time-average of the current expectation value $\left\langle I_{\ell}(t)\right\rangle$. Note that in a two-terminal device, the absolute value of the average current is independent of the contact $\ell$. The Fano factor is constructed such that for uncorrelated events $F=1$, i.e., it describes the noise levels with respect to a Poisson process. Historically, the zero-frequency noise (2.10) contains a factor of 2, i.e. $\bar{S}^{\prime}=2 \bar{S}$, resulting from a different definition of the Fourier transform. Then, the Fano factor is defined as $F=\bar{S}^{\prime} / 2 e|\bar{I}|$.

\subsection{Floquet Approach to the Driven Transport Problem}

We now derive from the model described in Sect. 2.2.1 expressions for both the current through the molecular wire and the associated noise by solving the corresponding Heisenberg equations of motions. To do so, we start from the equations of motion for the annihilation operators in lead $\ell$,

$$
\dot{c}_{\ell q}=-\frac{\mathrm{i}}{\hbar} \epsilon_{\ell q} c_{\ell q}-\frac{\mathrm{i}}{\hbar} V_{\ell q} c_{n_{\ell}},
$$

which are straightforwardly integrated to read

$$
c_{\ell q}(t)=c_{\ell q}\left(t_{0}\right) e^{-\mathrm{i} \epsilon_{\ell q}\left(t-t_{0}\right) / \hbar}-\frac{\mathrm{i}}{\hbar} V_{\ell q} \int_{0}^{t-t_{0}} \mathrm{~d} \tau e^{-\mathrm{i} \epsilon_{\ell q} \tau / \hbar} c_{n_{\ell}}(t-\tau),
$$

where $n_{\ell}$ denotes the molecular wire site attached to lead $\ell$, i.e., $n_{\mathrm{L}}=1$ and $n_{\mathrm{R}}=N$. Inserting (2.14) into the Heisenberg equations for the wire operators yields in the asymptotic limit $t_{0} \rightarrow-\infty$

$$
\begin{aligned}
\dot{c}_{n_{\ell}}(t) & =-\frac{\mathrm{i}}{\hbar} \sum_{n^{\prime}} H_{n_{\ell}, n^{\prime}}(t) c_{n^{\prime}}(t)-\frac{1}{\hbar} \int_{0}^{\infty} \mathrm{d} \tau \Gamma_{\ell}(\tau) c_{n_{\ell}}(t-\tau)+\xi_{\ell}(t), \\
\dot{c}_{n}(t) & =-\frac{\mathrm{i}}{\hbar} \sum_{n^{\prime}} H_{n n^{\prime}}(t) c_{n^{\prime}}(t), \quad n=2, \ldots, N-1,
\end{aligned}
$$

where the lead response function $\Gamma_{\ell}(t)$ results from the Fourier transformation of the spectral density (2.5),

$$
\Gamma_{\ell}(t)=\int_{-\infty}^{+\infty} \frac{\mathrm{d} \epsilon}{2 \pi \hbar} \mathrm{e}^{-\mathrm{i} \epsilon t / \hbar} \Gamma_{\ell}(\epsilon) .
$$

In the wide-band limit $\Gamma_{\ell}(\epsilon) \rightarrow \Gamma_{\ell}$, one obtains $\Gamma_{\ell}(t)=\Gamma_{\ell} \delta(t)$ and, thus, the equations of motion for the wire operators are memory-free. The influence of the operator-valued Gaussian noise

$$
\xi_{\ell}(t)=-\frac{\mathrm{i}}{\hbar} \sum_{q} V_{\ell q}^{*} e^{-\mathrm{i} \epsilon_{\ell q}\left(t-t_{0}\right) / \hbar} c_{\ell q}\left(t_{0}\right)
$$


is fully specified by the expectation values $\left\langle\xi_{\ell}(t)\right\rangle=0$ and

$$
\left\langle\xi_{\ell^{\prime}}^{\dagger}\left(t^{\prime}\right) \xi_{\ell}(t)\right\rangle=\delta_{\ell \ell^{\prime}} \frac{1}{2 \pi \hbar^{2}} \int \mathrm{d} \epsilon \mathrm{e}^{-\mathrm{i} \epsilon\left(t-t^{\prime}\right) / \hbar} \Gamma_{\ell}(\epsilon) f_{\ell}(\epsilon),
$$

which follow directly from the definition (18) and the initial conditions (2.6). It is convenient to define the Fourier representation of the noise operator, $\xi_{\ell}(\epsilon)=\int \mathrm{d} t \exp (\mathrm{i} \epsilon t / \hbar) \xi_{\ell}(t)$ whose correlation function

$$
\left\langle\xi_{\ell}^{\dagger}(\epsilon) \xi_{\ell^{\prime}}\left(\epsilon^{\prime}\right)\right\rangle=2 \pi \Gamma_{\ell}(\epsilon) f_{\ell}(\epsilon) \delta\left(\epsilon-\epsilon^{\prime}\right) \delta_{\ell \ell^{\prime}}
$$

follows directly from (2.19).

\subsubsection{Retarded Green Function}

The equations of motion (2.15) and (2.16) represent a set of linear inhomogeneous equations and, thus, can be solved with the help of a retarded Green function $G\left(t, t^{\prime}\right)=-(\mathrm{i} / \hbar) U\left(t, t^{\prime}\right) \theta\left(t-t^{\prime}\right)$, which obeys

$$
\left(\mathrm{i} \hbar \frac{\mathrm{d}}{\mathrm{d} t}-\mathcal{H}(t)\right) G\left(t, t^{\prime}\right)+\mathrm{i} \int_{0}^{\infty} \mathrm{d} \tau \Gamma(\tau) G\left(t-\tau, t^{\prime}\right)=\delta\left(t-t^{\prime}\right),
$$

where $\Gamma(t)=|1\rangle \Gamma_{\mathrm{L}}(t)\langle 1|+| N\rangle \Gamma_{\mathrm{R}}(t)\langle N|$ and $\mathcal{H}(t)$ is the one-particle Hamiltonian corresponding to (2.2). At this stage, it is important to note upon inspection that the propagator of the homogeneous equations obeys $U\left(t, t^{\prime}\right)=$ $U\left(t+\mathcal{T}, t^{\prime}+\mathcal{T}\right)$ and, accordingly, the Fourier representation of the retarded Green function

$$
G(t, \epsilon)=-\frac{\mathrm{i}}{\hbar} \int_{0}^{\infty} \mathrm{d} \tau \mathrm{e}^{\mathrm{i} \epsilon \tau / \hbar} U(t, t-\tau)=G(t+\mathcal{T}, \epsilon)
$$

is also $\mathcal{T}$-periodic in the time argument. Thus, we can employ the Fourier decomposition $G(t, \epsilon)=\sum_{k} \mathrm{e}^{-\mathrm{i} k \Omega t} G^{(k)}(\epsilon)$ with the coefficients

$$
G^{(k)}(\epsilon)=\frac{1}{\mathcal{T}} \int_{0}^{\mathcal{T}} \mathrm{d} t \mathrm{e}^{\mathrm{i} k \Omega t} G(t, \epsilon) .
$$

Physically, $G^{(k)}(\epsilon)$ describes the propagation of an electron with initial energy $\epsilon$ under the absorption (emission) of $|k|$ photons for $k>0(k<0)$. In the limiting case of a time-independent situation, all sideband contributions with $k \neq 0$ vanish and $G(t, \epsilon)$ becomes time-independent and identical to $G^{(0)}(\epsilon)$.

From the definition (2.21) of the Green function and its Fourier representation (2.22), it can be shown that the solution of the Heisenberg equations (2.15), (2.16) reads

$$
c_{n}(t)=\frac{\mathrm{i}}{2 \pi} \sum_{\ell} \int \mathrm{d} \epsilon \mathrm{e}^{-\mathrm{i} \epsilon t / \hbar} G_{n, n_{\ell}}(t, \epsilon) \xi_{\ell}(\epsilon),
$$


where we have defined $G_{n, n_{\ell}}\left(t, t^{\prime}\right)=\left\langle n\left|G\left(t, t^{\prime}\right)\right| n_{\ell}\right\rangle$.

Below, we need for the elimination of back-scattering terms the relation

$$
\begin{aligned}
G^{\dagger}\left(t, \epsilon^{\prime}\right)-G(t, \epsilon)= & \left(\mathrm{i} \hbar \frac{\mathrm{d}}{\mathrm{d} t}-\epsilon^{\prime}+\epsilon\right) G^{\dagger}\left(t, \epsilon^{\prime}\right) G(t, \epsilon) \\
& +\mathrm{i} \int_{0}^{\infty} \mathrm{d} \tau \mathrm{e}^{\mathrm{i} \epsilon \tau / \hbar} G^{\dagger}\left(t, \epsilon^{\prime}\right) \Gamma(\tau) G(t-\tau, \epsilon) \\
& +\mathrm{i} \int_{0}^{\infty} \mathrm{d} \tau \mathrm{e}^{-\mathrm{i} \epsilon^{\prime} \tau / \hbar} G^{\dagger}\left(t-\tau, \epsilon^{\prime}\right) \Gamma^{\dagger}(\tau) G(t, \epsilon) .
\end{aligned}
$$

A proof starts from the definition (2.21) of the Green function. By Fourier transformation with respect to $t^{\prime}$, we obtain the relation

$$
\left(\mathrm{i} \hbar \frac{\mathrm{d}}{\mathrm{d} t}+\epsilon-\mathcal{H}(t)\right) G(t, \epsilon)+\mathrm{i} \int_{0}^{\infty} \mathrm{d} \tau \mathrm{e}^{\mathrm{i} \epsilon \tau / \hbar} \Gamma(\tau) G(t-\tau, \epsilon)=\mathbf{1}
$$

which we multiply by $G^{\dagger}(t, \epsilon)$ from the left. The difference between the resulting expression and its hermitian adjoint with $\epsilon$ and $\epsilon^{\prime}$ interchanged is relation $(2.25)$.

\subsubsection{Current Through the Driven Molecular Wire}

Owing to charge conservation, the (net) current flowing from lead $\ell$ into the molecular wire is determined by the negative time derivative of the charge in lead $\ell$. Thus, the current operator reads $I_{\ell}=\mathrm{i} e\left[H(t), N_{\ell}\right] / \hbar$, where $N_{\ell}=$ $\sum_{q} c_{\ell q}^{\dagger} c_{\ell q}$ denotes the corresponding electron number and $-e$ the electron charge. By using (2.14) and (2.18), we obtain

$$
\begin{aligned}
I_{\mathrm{L}}(t)= & \frac{e}{\hbar} \int_{0}^{\infty} \mathrm{d} \tau\left\{\Gamma_{\mathrm{L}}(\tau) c_{1}^{\dagger}(t) c_{1}(t-\tau)+\Gamma_{\mathrm{L}}^{*}(\tau) c_{1}^{\dagger}(t-\tau) c_{1}(t)\right\} \\
& -e\left\{c_{1}^{\dagger}(t) \xi_{\mathrm{L}}(t)+\xi_{\mathrm{L}}^{\dagger}(t) c_{1}(t)\right\} .
\end{aligned}
$$

This operator-valued expression for the time-dependent current is a convenient starting point for the evaluation of expectation values like the dc and ac current and the current noise.

\section{Time-Average Current}

To obtain the current $\left\langle I_{\mathrm{L}}(t)\right\rangle$, we insert the solution (2.24) of the Heisenberg equation into the current operator (2.27) and use the expectation values (2.20). The resulting expression

$$
\begin{aligned}
\left\langle I_{\mathrm{L}}(t)\right\rangle= & \frac{2 e}{h} \sum_{\ell} \int \mathrm{d} \epsilon \int_{0}^{\infty} \mathrm{d} \tau \Gamma_{\ell}(\epsilon) f_{\ell}(\epsilon) \operatorname{Im} \mathrm{e}^{\mathrm{i} \epsilon \tau / \hbar} G_{1 \ell}^{*}(t, \epsilon) \Gamma_{\mathrm{L}}(\tau) G_{1 \ell}(t-\tau, \epsilon) \\
& +2 e \int \mathrm{d} \epsilon \Gamma_{\mathrm{L}}(\epsilon) f_{\mathrm{L}}(\epsilon) \operatorname{Im} G_{11}(t, \epsilon)
\end{aligned}
$$


still contains back-scattering terms $G_{11}$ and, thus, is not of a "scattering form". Indeed, bringing (2.28) into a form that resembles the static current formula (2.7) requires some tedious algebra. Such a derivation has been presented for the linear conductance of time-independent systems [30], for tunnelling barriers [29] and mesoscopic conductors [31] in the static case for finite voltage, and for a wire consisting of levels that couple equally strong to both leads [34]. For the periodically time-dependent case in the absence of electron-electron interactions, such an expression has been derived only recently $[35,36]$.

Inserting the matrix element $\langle 1|\ldots| 1\rangle$ of $(2.25)$ eliminates the backscattering terms and yields for the time-dependent current the expression

$$
\left\langle I_{\mathrm{L}}(t)\right\rangle=\frac{e}{h} \int \mathrm{d} \epsilon\left\{T_{\mathrm{LR}}(t, \epsilon) f_{\mathrm{R}}(\epsilon)-T_{\mathrm{RL}}(t, \epsilon) f_{\mathrm{L}}(\epsilon)\right\}-\frac{\mathrm{d}}{\mathrm{d} t} q_{\mathrm{L}}(t)
$$

where

$$
q_{\mathrm{L}}(t)=\frac{e}{2 \pi} \int \mathrm{d} \epsilon \Gamma_{\mathrm{L}}(\epsilon) \sum_{n}\left|G_{n 1}(t, \epsilon)\right|^{2} f_{\mathrm{L}}(\epsilon)
$$

denotes the charge oscillating between the left lead and the wire. Obviously, since $q_{\mathrm{L}}(t)$ is time-periodic and bounded, its time derivative cannot contribute to the average current. The corresponding charge arising from the right lead, $q_{\mathrm{R}}(t)$, is a priori unrelated to $q_{\mathrm{L}}(t)$; the actual charge on the wire reads $q_{\mathrm{L}}(t)+q_{\mathrm{R}}(t)$. The time-dependent current is determined by the time-dependent transmission

$$
T_{\mathrm{LR}}(t, \epsilon)=2 \operatorname{Re} \int_{0}^{\infty} \mathrm{d} \tau \mathrm{e}^{\mathrm{i} \epsilon \tau / \hbar} \Gamma_{\mathrm{L}}(\tau) G_{1 N}^{*}(t, \epsilon) G_{1 N}(t-\tau, \epsilon) \Gamma_{\mathrm{R}}(\epsilon) .
$$

The corresponding expression for $T_{\mathrm{RL}}(t, \epsilon)$ follows from the replacement $(\mathrm{L}, 1) \leftrightarrow(\mathrm{R}, N)$. We emphasise that (2.29) obeys the form of the current formula (2.7) for a static conductor within a scattering formalism. In particular, consistent with [37,38], no "Pauli blocking factors" $\left(1-f_{\ell}\right)$ appear in our derivation. In contrast to a static situation, this is in the present context relevant since for a driven system generally $T_{\mathrm{LR}}^{(k)}(\epsilon) \neq T_{\mathrm{RL}}^{(k)}(\epsilon)$, such that a contribution proportional to $f_{\mathrm{L}}(\epsilon) f_{\mathrm{R}}(\epsilon)$ would not cancel $[37,39]$.

The dc current obtained from (2.29) by time-averaging can be written in an even more compact form if we insert for the Green function the Fourier representation $(2.23)$. This results in

$$
\bar{I}=\frac{e}{h} \sum_{k} \int \mathrm{d} \epsilon\left\{T_{\mathrm{LR}}^{(k)}(\epsilon) f_{\mathrm{R}}(\epsilon)-T_{\mathrm{RL}}^{(k)}(\epsilon) f_{\mathrm{L}}(\epsilon)\right\},
$$

where

$$
\begin{aligned}
& T_{\mathrm{LR}}^{(k)}(\epsilon)=\Gamma_{\mathrm{L}}(\epsilon+k \hbar \Omega) \Gamma_{\mathrm{R}}(\epsilon)\left|G_{1 N}^{(k)}(\epsilon)\right|^{2}, \\
& T_{\mathrm{RL}}^{(k)}(\epsilon)=\Gamma_{\mathrm{R}}(\epsilon+k \hbar \Omega) \Gamma_{\mathrm{L}}(\epsilon)\left|G_{N 1}^{(k)}(\epsilon)\right|^{2},
\end{aligned}
$$


denote the transmission probabilities for electrons from the right to the left lead and vice versa, respectively, with initial energy $\epsilon$ and final energy $\epsilon+k \hbar \Omega$, i.e., the probability for an scattering event under the absorption (emission) of $|k|$ photons if $k>0(k<0)$.

For a static situation, the transmissions $T_{\mathrm{LR}}^{(k)}(\epsilon)$ and $T_{\mathrm{RL}}^{(k)}(\epsilon)$ are identical and contributions with $k \neq 0$ vanish. Thus, it is possible to write the current (2.32) in the form (2.7) as a product of a single transmission $T(\epsilon)$, which is independent of the direction, and the difference of the Fermi functions, $f_{\mathrm{R}}(\epsilon)-f_{\mathrm{L}}(\epsilon)$. We emphasise that in the driven case this no longer holds true.

\section{Noise Power}

Like in the static case, we characterise the noise power by the zero-frequency component of the current-current correlation function (2.9). However, in the driven case, $S_{\ell}\left(t, t^{\prime}\right)=S_{\ell}\left(t+\mathcal{T}, t^{\prime}+\mathcal{T}\right)$ is still time-dependent. Since it shares the time-periodicity of the driving, it is possible to characterise the noise level by the zero-frequency component of $S_{\ell}(t, t-\tau)$ averaged over the driving period,

$$
\bar{S}_{\ell}=\frac{1}{\mathcal{T}} \int_{0}^{\mathcal{T}} \mathrm{d} t \int_{-\infty}^{\infty} \mathrm{d} \tau S_{\ell}(t, t-\tau) .
$$

It can be shown [36] that for driven two-terminal devices, $\bar{S}_{\ell}$ is independent of the contact $\ell$, i.e., $\bar{S}_{\mathrm{L}}=\bar{S}_{\mathrm{R}} \equiv \bar{S}$.

We start by writing $S_{\mathrm{L}}(t, t-\tau)$ with the current operator (2.27) and insert the solution (2.24) of the Heisenberg equations of motion. Then, we again employ relation (2.25) and finally obtain the more symmetric expression

$$
\begin{aligned}
& \bar{S}=\frac{e^{2}}{h} \sum_{k} \int \mathrm{d} \epsilon\left\{\Gamma_{\mathrm{R}}\left(\epsilon_{k}\right) \Gamma_{\mathrm{R}}(\epsilon)\left|\sum_{k^{\prime}} \Gamma_{\mathrm{L}}\left(\epsilon_{k^{\prime}}\right) G_{1 N}^{\left(k^{\prime}-k\right)}\left(\epsilon_{k}\right)\left[G_{1 N}^{\left(k^{\prime}\right)}(\epsilon)\right]^{*}\right|^{2} f_{\mathrm{R}}(\epsilon) \bar{f}_{\mathrm{R}}\left(\epsilon_{k}\right)\right. \\
& \left.+\Gamma_{\mathrm{R}}\left(\epsilon_{k}\right) \Gamma_{\mathrm{L}}(\epsilon)\left|\sum_{k^{\prime}} \Gamma_{\mathrm{L}}\left(\epsilon_{k^{\prime}}\right) G_{1 N}^{\left(k^{\prime}-k\right)}\left(\epsilon_{k}\right)\left[G_{11}^{\left(k^{\prime}\right)}(\epsilon)\right]^{*}-\mathrm{i} G_{1 N}^{(-k)}\left(\epsilon_{k}\right)\right|^{2} f_{\mathrm{L}}(\epsilon) \bar{f}_{\mathrm{R}}\left(\epsilon_{k}\right)\right\} \\
& + \text { same terms with the replacement }(\mathrm{L}, 1) \leftrightarrow(\mathrm{R}, N) .
\end{aligned}
$$

We have defined $\epsilon_{k}=\epsilon+k \hbar \Omega$.

\section{Floquet Decomposition in the Wide-Band Limit}

We now consider the wide-band limit $\Gamma_{\ell}(\epsilon)=\Gamma_{\ell}$ and, consequently, $\Gamma_{\ell}(t)=$ $\Gamma_{\ell} \delta(\tau)$. Then, solving the equations of motion (2.21) for the Green function is equivalent to computing a complete set of solutions for the equation

$$
\mathrm{i} \hbar \frac{\mathrm{d}}{\mathrm{d} t}|\psi(t)\rangle=\left(\mathcal{H}_{\text {wire }}(t)-\mathrm{i} \Sigma\right)|\psi(t)\rangle
$$


with the self-energy $\Sigma=|1\rangle \Gamma_{\mathrm{L}} / 2\langle 1|+| N\rangle \Gamma_{\mathrm{R}} / 2\langle N|$ resulting from the coupling to the leads. Equation (2.37) is linear and possesses time-dependent, $\mathcal{T}$-periodic coefficients. Thus, it is possible to construct a complete set of solutions with the Floquet ansatz

$$
\begin{aligned}
\left|\psi_{\alpha}(t)\right\rangle & =\exp \left[\left(-\mathrm{i} \epsilon_{\alpha} / \hbar-\gamma_{\alpha}\right) t\right]\left|u_{\alpha}(t)\right\rangle \\
\left|u_{\alpha}(t)\right\rangle & =\sum_{k} \exp (-\mathrm{i} k \Omega t)\left|u_{\alpha, k}\right\rangle
\end{aligned}
$$

The so-called Floquet states $\left|u_{\alpha}(t)\right\rangle$ obey the time-periodicity of $\mathcal{H}_{\text {wire }}(t)$ and have been decomposed into a Fourier series. In a Hilbert space that is extended by a periodic time coordinate, the so-called Sambe space [40], they obey the Floquet eigenvalue equation $[41,42]$

$$
\left(\mathcal{H}_{\text {wire }}(t)-\mathrm{i} \Sigma-\mathrm{i} \hbar \frac{\mathrm{d}}{\mathrm{d} t}\right)\left|u_{\alpha}(t)\right\rangle=\left(\epsilon_{\alpha}-\mathrm{i} \hbar \gamma_{\alpha}\right)\left|u_{\alpha}(t)\right\rangle .
$$

Due to the Brillouin zone structure of the Floquet spectrum $[40,41,43]$, it is sufficient to compute all eigenvalues of the first Brillouin zone, $-\hbar \Omega / 2<\epsilon_{\alpha} \leq$ $\hbar \Omega / 2$. Since the operator on the l.h.s. of (2.40) is non-Hermitian, the eigenvalues $\epsilon_{\alpha}-\mathrm{i} \hbar \gamma_{\alpha}$ are generally complex-valued and the (right) eigenvectors are not mutually orthogonal. Thus, to determine the propagator, we need to solve also the adjoint Floquet equation yielding again the same eigenvalues but providing the adjoint eigenvectors $\left|u_{\alpha}^{+}(t)\right\rangle$. It can be shown that the Floquet states $\left|u_{\alpha}(t)\right\rangle$ together with the adjoint states $\left|u_{\alpha}^{+}(t)\right\rangle$ form at equal times a complete bi-orthogonal basis: $\left\langle u_{\alpha}^{+}(t) \mid u_{\beta}(t)\right\rangle=\delta_{\alpha \beta}$ and $\sum_{\alpha}\left|u_{\alpha}(t)\right\rangle\left\langle u_{\alpha}^{+}(t)\right|=\mathbf{1}$. A proof requires to account for the time-periodicity of the Floquet states since the eigenvalue equation (2.40) holds in a Hilbert space extended by a periodic time coordinate $[41,44]$.

Using the Floquet equation (2.40), it is straightforward to show that the propagator can be written as

$$
U\left(t, t^{\prime}\right)=\sum_{\alpha} \mathrm{e}^{-\mathrm{i}\left(\epsilon_{\alpha} / \hbar-\mathrm{i} \gamma_{\alpha}\right)\left(t-t^{\prime}\right)}\left|u_{\alpha}(t)\right\rangle\left\langle u_{\alpha}^{+}\left(t^{\prime}\right)\right|
$$

where the sum runs over all Floquet states within one Brillouin zone. Consequently, the Fourier coefficients of the Green function read

$$
\begin{aligned}
G^{(k)}(\epsilon) & =-\frac{\mathrm{i}}{\hbar} \int_{0}^{\mathcal{T}} \frac{\mathrm{d} t}{\mathcal{T}} e^{\mathrm{i} k \Omega t} \int_{0}^{\infty} \mathrm{d} \tau \mathrm{e}^{\mathrm{i} \epsilon \tau / \hbar} U(t, t-\tau) \\
& =\sum_{\alpha, k^{\prime}} \frac{\left|u_{\alpha, k^{\prime}+k}\right\rangle\left\langle u_{\alpha, k^{\prime}}^{+}\right|}{\epsilon-\left(\epsilon_{\alpha}+k^{\prime} \hbar \Omega-\mathrm{i} \hbar \gamma_{\alpha}\right)} .
\end{aligned}
$$

In general, the Floquet equation (2.40) has to be solved numerically. In the zero temperature limit considered here, the Fermi functions in the expressions for the average current (2.32) and the zero-frequency noise (2.36) become step functions. Therefore, the integrands are rational functions and the remaining energy integrals can be performed analytically. 


\subsection{Weak-Coupling Approximations}

In the previous section, exact expression for the dc current and the zerofrequency noise for a periodic but otherwise arbitrary ac driving have been derived. Within the wide-band limit, both quantities can be expressed in terms of the solutions of the Floquet equation (2.40), i.e., the solution of a non-Hermitian eigenvalue problem in an extended Hilbert space. For the case of energy-dependent wire-lead coupling $\Gamma_{\ell}(\epsilon)$, the corresponding computation becomes rather cumbersome. Moreover, for finite temperatures, the energy integration in the expressions (2.32) and (2.36) has to be performed numerically. In the next two sections, we shall demonstrate how these problems can be overcome in the important special case of a weak wire-lead coupling.

\subsubsection{Asymptotically Weak Coupling}

In the limit of very weak wire-lead coupling, i.e., for coupling constants $\Gamma_{\ell}$ which are far lower than all other energy scales of the wire Hamiltonian, it is possible to treat the self-energy contribution $-\mathrm{i} \Sigma$ in the non-Hermitian Floquet equation (2.40) as perturbation. Then, the zeroth order of the Floquet equation

$$
\left(\mathcal{H}_{\text {wire }}(t)-\mathrm{i} \hbar \frac{\mathrm{d}}{\mathrm{d} t}\right)\left|\phi_{\alpha}(t)\right\rangle=\epsilon_{\alpha}^{0}\left|\phi_{\alpha}(t)\right\rangle,
$$

describes the driven wire in the absence of the leads, where $\left|\phi_{\alpha}(t)\right\rangle=$ $\sum_{k} \exp (-\mathrm{i} k \Omega t)\left|\phi_{\alpha, k}\right\rangle$ are the "usual" Floquet states with quasienergies $\epsilon_{\alpha}^{0}$. In the absence of degeneracies, the first order correction to the quasienergies is $-\mathrm{i} \hbar \gamma_{\alpha}^{1}$, where

$$
\begin{aligned}
\gamma_{\alpha}^{1} & =\frac{1}{\hbar} \int_{0}^{\mathcal{T}} \frac{\mathrm{d} t}{\mathcal{T}}\left\langle\phi_{\alpha}(t)|\Sigma| \phi_{\alpha}(t)\right\rangle \\
& =\frac{\Gamma_{\mathrm{L}}}{2 \hbar} \sum_{k}\left|\left\langle 1 \mid \phi_{\alpha, k}\right\rangle\right|^{2}+\frac{\Gamma_{\mathrm{R}}}{2 \hbar} \sum_{k}\left|\left\langle N \mid \phi_{\alpha, k}\right\rangle\right|^{2} .
\end{aligned}
$$

Since the first order correction to the Floquet states will contribute to neither the current nor the noise, the zeroth-order contribution $\left|u_{\alpha}(t)\right\rangle=\left|u_{\alpha}^{+}(t)\right\rangle=$ $\left|\phi_{\alpha}(t)\right\rangle$ is already sufficient for the present purpose. Consequently, the transmission (2.33) assumes the form

$$
T_{\mathrm{LR}}^{(k)}(\epsilon)=\Gamma_{\mathrm{L}} \Gamma_{\mathrm{R}} \sum_{\alpha, \beta, k^{\prime}, k^{\prime \prime}} \frac{\left\langle N \mid \phi_{\alpha, k^{\prime}}\right\rangle\left\langle\phi_{\alpha, k^{\prime}+k} \mid 1\right\rangle\left\langle 1 \mid \phi_{\beta, k^{\prime \prime}+k}\right\rangle\left\langle\phi_{\beta, k^{\prime \prime}} \mid N\right\rangle}{\left[\epsilon-\left(\epsilon_{\alpha}^{0}+k^{\prime} \hbar \Omega+\mathrm{i} \hbar \gamma_{\alpha}^{1}\right)\right]\left[\epsilon-\left(\epsilon_{\beta}^{0}+k^{\prime \prime} \hbar \Omega-\mathrm{i} \hbar \gamma_{\beta}^{1}\right)\right]}
$$

and $T_{\mathrm{RL}}^{(k)}(\epsilon)$ accordingly. The transmission (2.49) exhibits for small values of $\Gamma_{\ell}$ sharp peaks at energies $\epsilon_{\alpha}^{0}+k^{\prime} \hbar \Omega$ and $\epsilon_{\beta}^{0}+k^{\prime \prime} \hbar \Omega$ with widths $\hbar \gamma_{\alpha}^{1}$ and $\hbar \gamma_{\beta}^{1}$. Therefore, the relevant contributions to the sum come from terms for 
which the peaks of both factors coincide and, in the absence of degeneracies in the quasienergy spectrum, we keep only terms with

$$
\alpha=\beta, \quad k^{\prime}=k^{\prime \prime} .
$$

Then provided that $\gamma_{\alpha}^{1}$ is small, the fraction in (2.49) is a Lorentzian and can be approximated by $\pi \delta\left(\epsilon-\epsilon_{\alpha}^{0}-k^{\prime} \hbar \Omega\right) / \hbar \gamma_{\alpha}^{1}$ yielding the transmission

$$
T_{\mathrm{LR}}^{(k)}(\epsilon)=\Gamma_{\mathrm{L}} \Gamma_{\mathrm{R}} \sum_{\alpha, k^{\prime}} \frac{\pi}{\hbar \gamma_{\alpha}^{1}}\left|\left\langle 1 \mid \phi_{\alpha, k^{\prime}+k}\right\rangle\left\langle\phi_{\alpha, k^{\prime}} \mid N\right\rangle\right|^{2} \delta\left(\epsilon-\epsilon_{\alpha}^{0}+k^{\prime} \hbar \Omega\right) .
$$

The energy integration in (2.32) can now be performed even for finite temperature and we obtain for the dc current the expression

$$
\bar{I}=\frac{e}{\hbar} \sum_{\alpha, k, k^{\prime}} \frac{\Gamma_{\mathrm{L} \alpha k} \Gamma_{\mathrm{R} \alpha k^{\prime}}}{\Gamma_{\mathrm{L} \alpha}+\Gamma_{\mathrm{R} \alpha}}\left[f_{\mathrm{R}}\left(\epsilon_{\alpha}^{0}+k^{\prime} \hbar \Omega\right)-f_{\mathrm{L}}\left(\epsilon_{\alpha}^{0}+k \hbar \Omega\right)\right] .
$$

The coefficients

$$
\begin{array}{ll}
\Gamma_{\mathrm{L} \alpha k}=\Gamma_{\mathrm{L}}\left|\left\langle 1 \mid \phi_{\alpha, k}\right\rangle\right|^{2}, & \Gamma_{\mathrm{L} \alpha}=\sum_{k} \Gamma_{\mathrm{L} \alpha k}, \\
\Gamma_{\mathrm{R} \alpha k}=\Gamma_{\mathrm{R}}\left|\left\langle N \mid \phi_{\alpha, k}\right\rangle\right|^{2}, & \Gamma_{\mathrm{R} \alpha}=\sum_{k} \Gamma_{\mathrm{R} \alpha k},
\end{array}
$$

denote the overlap of the $k$ th sideband $\left|\phi_{\alpha, k}\right\rangle$ of the Floquet state $\left|\phi_{\alpha}(t)\right\rangle$ with the first site and the last site of the wire, respectively. We have used $2 \hbar \gamma_{\alpha}^{1}=\Gamma_{\mathrm{L} \alpha}+\Gamma_{\mathrm{R} \alpha}$ which follows from (2.46).

Within the same approximation, we expand the zero-frequency noise (2.36) to lowest-order in $\Gamma_{\ell}$ : After inserting the spectral representation (2.43) of the Green function, we again keep only terms with identical Floquet index $\alpha$ and identical sideband index $k$ to obtain

$$
\begin{aligned}
\bar{S}= & \frac{e^{2}}{\hbar} \sum_{\alpha, k, k^{\prime}} \frac{\Gamma_{\mathrm{R} \alpha k^{\prime}} \bar{f}_{\mathrm{R}}\left(\epsilon_{\alpha}^{0}+k^{\prime} \hbar \Omega\right)}{\left(\Gamma_{\mathrm{L} \alpha}+\Gamma_{\mathrm{R} \alpha}\right)^{3}}\left\{2 \Gamma_{\mathrm{L} \alpha}^{2} \Gamma_{\mathrm{R} \alpha k} f_{\mathrm{R}}\left(\epsilon_{\alpha}^{0}+k \hbar \Omega\right)\right. \\
& \left.+\left(\Gamma_{\mathrm{L} \alpha}^{2}+\Gamma_{\mathrm{R} \alpha}^{2}\right) \Gamma_{\mathrm{L} \alpha k} f_{\mathrm{L}}\left(\epsilon_{\alpha}^{0}+k \hbar \Omega\right)\right\} \\
& + \text { same terms with the replacement } L \leftrightarrow R
\end{aligned}
$$

\subsubsection{Master-Equation Approach}

An essential step in the derivation of the weak-coupling approximation (2.49) was the assumption that only terms with $\alpha=\beta$ contribute significantly to the transmission (2.47). This condition is clearly violated in the presence of degeneracies in the quasienergy spectrum or intermediate wire-lead coupling strength. Then, one has to refine the discussion presented above. 


\section{Current Formula}

We start again from the unsymmetric expression (2.28) for the timedependent current across the contact between the wire and the left lead. After performing the average over one driving period, it assumes the form

$$
\begin{aligned}
\bar{I}= & \frac{2 e}{h \mathcal{T}} \sum_{\ell} \int \mathrm{d} \epsilon \int_{0}^{\infty} \mathrm{d} \tau \int_{0}^{\mathcal{T}} \mathrm{d} t \Gamma_{\ell}(\epsilon) f_{\ell}(\epsilon) \operatorname{Im} \mathrm{e}^{\mathrm{i} \epsilon \tau / \hbar} G_{1 \ell}^{*}(t, \epsilon) \Gamma_{\mathrm{L}}(\tau) G_{1 \ell}(t-\tau, \epsilon) \\
& +2 e \int \mathrm{d} \epsilon \Gamma_{\mathrm{L}}(\epsilon) f_{\mathrm{L}}(\epsilon) \operatorname{Im} G_{11}^{(0)}(\epsilon) .
\end{aligned}
$$

Let us first consider the second term in this expression, which is linear in the retarded Green function $G_{11}^{(0)}(\epsilon)$ and, thus, its evaluation does not suffer from having two poles close to each other. Then, we can perform the energy integration in (2.54) the same way as in the last subsection to obtain the contribution

$$
-\frac{e}{\hbar} \sum_{\alpha, k}\left|\left\langle 1 \mid \phi_{\alpha, k}\right\rangle\right|^{2} \Gamma_{\mathrm{L}}\left(\epsilon_{\alpha}^{0}+k \hbar \Omega\right) f\left(\epsilon_{\alpha}^{0}+k \hbar \Omega-\mu_{\mathrm{L}}\right)
$$

to the time-averaged current.

The first term in (2.54) has to be treated with more care since it is quadratic in the retarded Green function. Thus, in the case of degeneracies of the quasienergy spectrum, the approximation scheme described in the previous paragraph breaks down (cf. also discussion in Sect. 2.4.1) and a more elaborate treatment is necessary. For this, it turns out to be advantageous to go one step back in the derivation of (2.54), namely to use the form given in (2.27), yielding for the first term in (2.54)

$$
\frac{2 e}{\hbar \mathcal{T}} \int_{0}^{\infty} \mathrm{d} \tau \int_{0}^{\mathcal{T}} \mathrm{d} t \operatorname{Re}\left[\Gamma_{\mathrm{L}}(\tau)\left\langle c_{1}^{\dagger}(t) c_{1}(t-\tau)\right\rangle\right] .
$$

Assuming that $\Gamma_{\mathrm{L}}(\epsilon)$ is a slowly varying function for all relevant energies $\epsilon$, i.e., $\Delta \Gamma_{\mathrm{L}} / \Gamma_{\mathrm{L}} \ll 1$, we can replace the time-evolution of $c_{1}$ from the time $t$ back to $t-\tau$ by the zeroth-order expression $c_{1}(t-\tau, t)=U_{0}^{\dagger}(t-\tau, t) c_{1} U_{0}(t-$ $\tau, t)$ with $U_{0}$ being the propagator $(2.41)$ in the limit $\Gamma_{\mathrm{L} / \mathrm{R}} \rightarrow 0$. We have introduced the wire operators $c_{\alpha}(t)$ in the "Floquet picture" by means of the time-dependent transformation $[24,25]$

$$
c_{\alpha}(t)=\sum_{n}\left\langle\phi_{\alpha}(t) \mid n\right\rangle c_{n} .
$$

Using the inverse transformation $c_{n}=\sum_{\alpha}\left\langle n \mid \phi_{\alpha}(t)\right\rangle c_{\alpha}(t)$, we obtain

$$
c_{1}(t-\tau, t) \approx \sum_{\alpha k} \mathrm{e}^{-\mathrm{i} k \Omega t} \mathrm{e}^{\mathrm{i}\left(\epsilon_{\alpha}^{0}+k \hbar \Omega\right) \tau / \hbar}\left\langle 1 \mid \phi_{\alpha, k}\right\rangle c_{\alpha}(t)
$$


such that we can then rewrite the current (2.56) in terms of the expectation values $P_{\alpha \beta}(t)=\left\langle c_{\beta}^{\dagger}(t) c_{\alpha}(t)\right\rangle_{t}$ at time $t$. In order to determine the values of $P_{\alpha \beta}(t)$ at asymptotic times, we now derive for these expectation values a kinetic equation. Before doing so, however, we simplify (2.56) further by using of the fact that the $P_{\alpha \beta}(t)$ are $\mathcal{T}$-periodic functions and can thus be decomposed into a Fourier series $P_{\alpha \beta}(t)=\sum_{k} \exp (-\mathrm{i} k \Omega t) P_{\alpha \beta, k}$. This brings (2.56) into the form

$$
\frac{2 e}{\hbar} \sum_{\alpha, \beta, k, k^{\prime}} \int_{0}^{\infty} \mathrm{d} \tau \operatorname{Re}\left[\Gamma_{\mathrm{L}}(\tau) \mathrm{e}^{\mathrm{i}\left(\epsilon_{\alpha}^{0}+k \hbar \Omega\right) \tau / \hbar}\left\langle\phi_{\beta, k+k^{\prime}} \mid 1\right\rangle\left\langle 1 \mid \phi_{\alpha, k}\right\rangle P_{\alpha \beta, k^{\prime}}\right] .
$$

Using the definition (2.17) of the Fourier transform of the spectral density $\Gamma_{\mathrm{L}}(\epsilon)$, we finally find for the time-averaged current through the molecular wire

$$
\begin{aligned}
\bar{I}=\frac{e}{\hbar} \sum_{\alpha, k} \Gamma_{\mathrm{L}}\left(\epsilon_{\alpha}^{0}+k \hbar \Omega\right)[ & \sum_{\beta, k^{\prime}} \operatorname{Re}\left(\left\langle\phi_{\beta, k+k^{\prime}} \mid 1\right\rangle\left\langle 1 \mid \phi_{\alpha, k}\right\rangle P_{\alpha \beta, k^{\prime}}\right) \\
& \left.-\left|\left\langle 1 \mid \phi_{\alpha, k}\right\rangle\right|^{2} f\left(\epsilon_{\alpha}^{0}+k \hbar \Omega-\mu_{\mathrm{L}}\right)\right] .
\end{aligned}
$$

Note that we have disregarded principal value terms corresponding to an energy renormalisation due to the wire-lead coupling.

\section{Floquet-Markov Master Equation}

Having expressed the current in terms of the wire expectation values $P_{\alpha \beta}(t)$, we now derive their dynamics in the regime of a weak to moderately strong wire-lead coupling. We thus consider the time-derivative $\dot{P}_{\alpha \beta}(t)$, which with the help of the zeroth-order Floquet equation (2.44) can be written as

$$
\dot{P}_{\alpha \beta}(t)=-\frac{\mathrm{i}}{\hbar}\left(\epsilon_{\alpha}^{0}-\epsilon_{\beta}^{0}\right) P_{\alpha \beta}(t)+\operatorname{Tr}\left[c_{\beta}^{\dagger}(t) c_{\alpha}(t) \dot{\rho}(t)\right] .
$$

In order to focus on the wire-lead coupling, we switch for the further evaluation of the second term to the corresponding interaction picture $\tilde{O}\left(t, t_{0}\right)=$ $U_{0}\left(t, t_{0}\right)^{\dagger} O(t) U_{0}\left(t, t_{0}\right)$, where the propagator of the isolated molecule and the leads can be formally written as

$$
U_{0}\left(t, t_{0}\right)=\overleftarrow{T} \exp \left(-\frac{\mathrm{i}}{\hbar} \int_{t_{0}}^{t} \mathrm{~d} t^{\prime}\left[H_{\text {wire }}\left(t^{\prime}\right)+H_{\text {leads }}\right]\right)
$$

The dynamics is then governed by the Liouville-von Neumann equation in the interaction picture

$$
\mathrm{i} \hbar \frac{\mathrm{d}}{\mathrm{d} t} \tilde{\varrho}\left(t, t_{0}\right)=\left[\widetilde{H}_{\text {contacts }}\left(t, t_{0}\right), \tilde{\varrho}\left(t, t_{0}\right)\right],
$$

which after integration reads 


$$
\tilde{\varrho}\left(t, t_{0}\right)=\tilde{\varrho}\left(t_{0}, t_{0}\right)-\frac{\mathrm{i}}{\hbar} \int_{t_{0}}^{t} \mathrm{~d} t^{\prime}\left[\widetilde{H}_{\text {contacts }}\left(t^{\prime}, t_{0}\right), \tilde{\varrho}\left(t^{\prime}, t_{0}\right)\right] \text {. }
$$

Upon re-insertion of this relation into the right-hand side of (2.63), we obtain an integro-differential equation for the time-derivative of the density matrix, namely

$$
\begin{aligned}
\dot{\widetilde{\varrho}}\left(t, t_{0}\right)= & -\frac{\mathrm{i}}{\hbar}\left[\widetilde{H}_{\text {contacts }}\left(t, t_{0}\right), \widetilde{\varrho}\left(t_{0}, t_{0}\right)\right] \\
& -\frac{1}{\hbar^{2}} \int_{0}^{t-t_{0}} \mathrm{~d} \tau\left[\widetilde{H}_{\text {contacts }}\left(t, t_{0}\right),\left[\widetilde{H}_{\text {contacts }}\left(t-\tau, t_{0}\right), \widetilde{\varrho}\left(t-\tau, t_{0}\right)\right]\right] .
\end{aligned}
$$

At this point, we assume that the dynamics induced by transitions due to the weak coupling $H_{\text {contacts }}$ is slow, so that $\widetilde{\varrho}\left(t-\tau, t_{0}\right)$ can be approximated by $\widetilde{\varrho}\left(t, t_{0}\right)$ in the integral. This so-called Born-Markov-approximation of course also requires $t \gg t_{0}$. After a back transformation into the Schrödinger picture, we therefore consider the asymptotic limit $t_{0} \rightarrow-\infty$. Using the resulting expression, we can cast (2.61) into the form

$$
\begin{aligned}
\dot{P}_{\alpha \beta}(t)= & -\frac{\mathrm{i}}{\hbar}\left(\epsilon_{\alpha}^{0}-\epsilon_{\beta}^{0}\right) P_{\alpha \beta}(t) \\
& -\frac{1}{\hbar^{2}} \int_{0}^{\infty} \mathrm{d} \tau\left\langle\left[\left[c_{\beta}^{\dagger}(t) c_{\alpha}(t), H_{\text {contacts }}\right], \widetilde{H}_{\text {contacts }}(t-\tau, t)\right]\right\rangle_{t} .
\end{aligned}
$$

Here, we have used twice the relation $\operatorname{Tr} A[B, C]=\operatorname{Tr}[A, B] C$, which directly results from the cyclic invariance of the trace. For the further evaluation of $H_{\text {contacts }}(t-\tau, t)$, we use that $\tilde{c}_{n}(t-\tau, t)$ is given by the right-hand side of (2.58). After some algebraic manipulations of the double commutator in the integrand, we eventually arrive at the Floquet-Markov master equation

$$
\begin{aligned}
\dot{P}_{\alpha \beta}= & -\frac{\mathrm{i}}{\hbar}\left(\epsilon_{\alpha}^{0}-\epsilon_{\beta}^{0}\right) P_{\alpha \beta}+\frac{1}{2} \sum_{\ell=\mathrm{L}, \mathrm{R}} \sum_{k k^{\prime}} \mathrm{e}^{\mathrm{i}\left(k^{\prime}-k\right) \Omega t} \\
\times\{ & \Gamma_{\ell}\left(\epsilon_{\alpha}^{0}+k \hbar \Omega\right)\left\langle\phi_{\alpha, k^{\prime}} \mid n_{\ell}\right\rangle\left\langle n_{\ell} \mid \phi_{\beta, k}\right\rangle f\left(\epsilon_{\alpha}^{0}+k \hbar \Omega-\mu_{\ell}\right) \\
& +\Gamma_{\ell}\left(\epsilon_{\beta}^{0}+k \hbar \Omega\right)\left\langle\phi_{\alpha, k^{\prime}} \mid n_{\ell}\right\rangle\left\langle n_{\ell} \mid \phi_{\beta, k}\right\rangle f\left(\epsilon_{\beta}^{0}+k \hbar \Omega-\mu_{\ell}\right) \\
& -\sum_{\alpha^{\prime}} \Gamma_{\ell}\left(\epsilon_{\alpha^{\prime}}^{0}+k \hbar \Omega\right)\left\langle\phi_{\alpha, k^{\prime}} \mid n_{\ell}\right\rangle\left\langle n_{\ell} \mid \phi_{\alpha^{\prime}, k}\right\rangle P_{\alpha^{\prime} \beta} \\
& \left.-\sum_{\beta^{\prime}} \Gamma_{\ell}\left(\epsilon_{\beta^{\prime}}^{0}+k^{\prime} \hbar \Omega\right)\left\langle\phi_{\beta^{\prime}, k^{\prime}} \mid n_{\ell}\right\rangle\left\langle n_{\ell} \mid \phi_{\beta, k}\right\rangle P_{\alpha \beta^{\prime}}\right\} .
\end{aligned}
$$

Here, we have assumed that the ideal leads always stay in thermal equilibrium and thus are described by the expectation values (2.6). Moreover, principal 
value terms stemming from an renormalisation of the wire energies due to the coupling to the leads have been neglected again.

The solution of the master equation together with the current expression (2.60) derived earlier, permits an efficient numerical calculation of the dc current through the molecular wire even for rather large systems or for energy-dependent couplings. Furthermore, as we shall demonstrate later on the basis of a specific example, this approach still works well in the presence of degeneracies in the quasienergy spectrum. In fact, one can recover the weak-coupling expansion presented in Sect. 2.4.1 from the master equation formalism within a so-called rotating-wave approximation [25]. Finally, we note that the master equation (2.67) can be generalised to describe within a mean-field description also the influence of an additional electron-vibrational coupling on the molecular wire [45].

\subsection{Photon-Assisted Transport Across a Molecular Bridge}

As an application of our formalism, we now consider the photon-assisted transport through a molecular bridge as sketched in Fig. 2.1. In our numerical studies, we use the hopping matrix element $\Delta$ as the energy unit; in a realistic molecule, $\Delta$ is of the order $0.1 \mathrm{eV}$. We choose a rather small wire-lead hopping rate $\Gamma=0.1 \Delta / \hbar$, which corresponds to a current $e \Gamma=2.56 \times 10^{-6}$ A. Laser frequencies $\Omega \approx \Delta / \hbar$ then lie in the infrared spectral range. For a typical distance of $5 \AA$ between two neighbouring sites, a driving amplitude $A=\Delta$ is equivalent to an electrical field strength of $2 \times 10^{6} \mathrm{~V} / \mathrm{cm}$.

It is instructive to first consider the situation without laser driving. Then in the limit $E_{\mathrm{B}} \gg \Delta$, transport through the donor-bridge-acceptor complex proceeds via the tunnel doublet $(|1\rangle \pm|N\rangle) / \sqrt{2}$ formed by the states $|1\rangle$ and $|N\rangle$. The tunnel coupling is mediated by the so-called super-exchange by way of the $N-2$ bridge states. The corresponding tunnel splitting is approximatively given by $2 \Delta\left(\Delta / E_{\mathrm{B}}\right)^{N-2}$ and thus is exponentially small [46]. For details, see Chap. 1.

When a laser field with a frequency that matches the energy difference between the tunnel doublet and the bridge states whose energies range from $E_{\mathrm{B}}-2 \Delta$ to $E_{\mathrm{B}}+2 \Delta$ is turned on, a direct channel for the transport through the wire is opened. At the resonance peaks, the current no longer decays exponentially fast with increasing wire length, but scales as $\bar{I} \propto A^{2} /(N-1) \Gamma[28]$. This photon-assisted tunnelling effect is shown in Fig. 2.2a, where the dc current is depicted as a function of the laser frequency $\Omega$. Both the results from an exact numerical calculation based on the scattering formula (2.32) and from the master equation formalism are displayed. They agree very well over the whole range of driving frequencies $\Omega$ as can be expected for resonant transport through the donor-bridge-acceptor complex. Due to the 


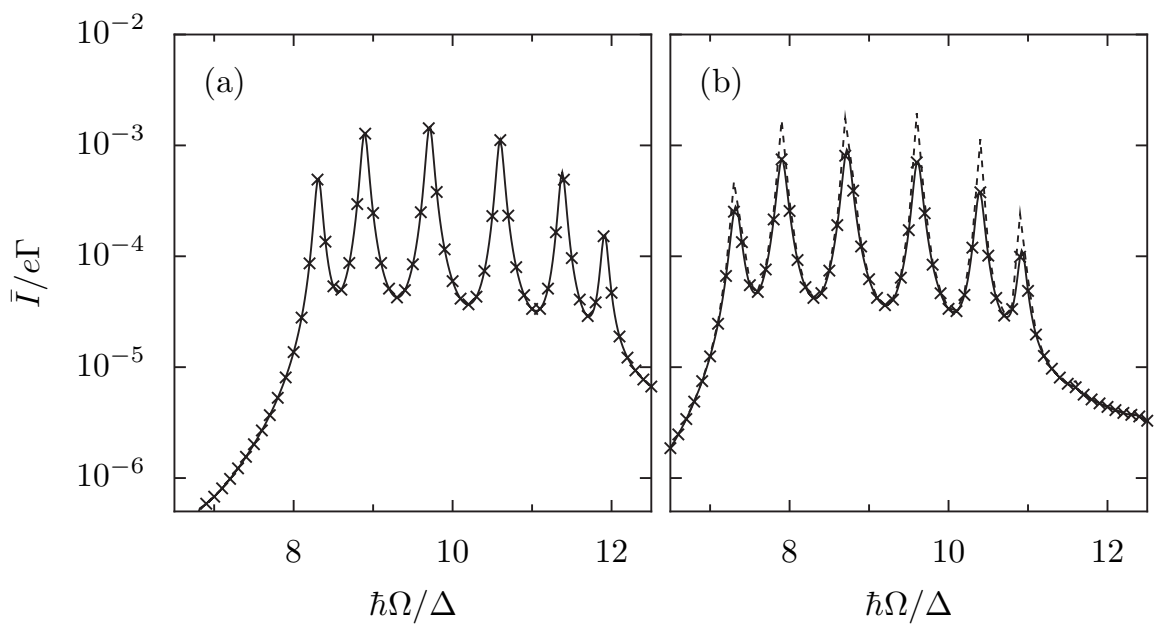

Fig. 2.2. Dc current $\bar{I}$ through molecular wire bridge with $N=8$ sites in the presence of a weak external driving with amplitude $A=0.1 \Delta$ as a function of the driving frequency $\Omega$. The bridge height is $E_{\mathrm{B}}=10 \Delta$ and the leftmost and the rightmost site lie at (a) $E_{1}=E_{8}=0$ and (b) $E_{1}=-\Delta$ and $E_{8}=\Delta$. The external bias voltage is $V=10 \Delta / e$, and the temperature $T=0$. The solid line shows the exact result (2.32) and the crosses correspond to the master equation result (2.60). The dashed line in panel (b) depicts the limit of very weak wire-lead coupling, (2.50)

quasi-degeneracy of the donor-acceptor doublet, the weak-coupling approximation (2.50) breaks down completely in the present case and incorrectly predicts a current $\bar{I}=e \Gamma / 2$ independent of the driving frequency $\Omega$ (not shown).

Due to possible asymmetries of the molecule itself or as a consequence of the applied dc bias voltage, the assumption that the donor and the acceptor levels are degenerate, might not be fulfilled. Thus, it is desirable to test the stability of the above results against shifts of the on-site energies of the donor and the acceptor. The dc current for this situation is shown in Fig. 2.2b. We again observe that the master equation result agrees very well with the numerically exact result from the scattering formalism. Moreover, now also the weak-coupling approximation (2.50) agrees qualitatively with the exact results. However, the weak-coupling approximation fails to quantitatively predict the peak heights.

Figure 2.3 demonstrates that at the resonances, the Fano factor assumes values considerably lower than one as expected for the transport through a resonant single level [32]. This reveals that at the resonant excitations, the transport mechanism is no longer dominated by tunnelling across a high barrier. 


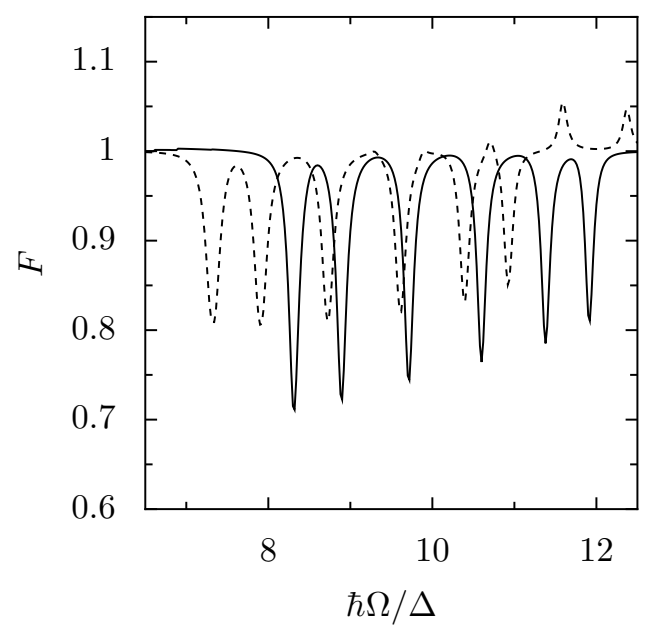

Fig. 2.3. Relative noise power characterised by the Fano factor $F=\bar{S} / e|\bar{I}|$ for unbiased (solid line) and biased (broken) donor and acceptor sites. All parameters are as in Fig. 2.2

\subsection{Conclusions}

We have put forward two theoretical approaches for the calculation of the dc current and its noise power through a molecular wire in the presence of a time-periodic external field, namely (i) a scattering approach and (ii) a master equation method. Both are based on the Floquet theorem and, thus, allow an exact treatment of the driven transport problem. The presented Floquet scattering formalism starts by integrating out the lead degrees of freedom. Then, the resulting equation of motion for the Green function of the wire electrons is mapped to a non-hermitian eigenvalue problem in a Hilbert space which is extended by a periodic time-coordinate. Finally, the expression for the dc current can be written in terms of generalised transmissions which describe electron transport under the absorption and emission of photons. The corresponding expression for the current noise, however, cannot be brought into such a convenient form: Indeed, in the presence of a time-dependent driving, the noise not only depends on transmission probabilities but also on the phases of the transmission amplitudes.

Alternatively, for sufficiently weak wire-lead coupling, the transport can be described by a master equation which is decomposed into the Floquet states of the isolated driven molecule. While being perturbative, this approach has also its benefits: Since only the Floquet states in the absence of the leads are needed, one can employ many standard methods known from the Floquet treatment of atoms and molecules in laser fields. Moreover, the master equation formalism can be extended readily to the case of energy-dependent couplings $\Gamma_{\ell}(\epsilon)$ and also allows one to include phonon-damping [45]. Finally, 
even for intermediately strong wire-lead coupling, the results obtained within the master equation approach agree very well with the exact results.

Of foremost interest in view of ongoing experiments, is the enhancement of molecular conduction by resonant excitations. The corresponding theoretical analysis has revealed that resonant excitations from the terminating atoms to the bridge levels yield peaks in the current as a function of the driving frequency. The laser irradiation induces a large current enhancement of several orders of magnitude and also can reduce the current noise level. The observation of these resonances could serve as an experimental starting point for the more challenging attempt of measuring quantum ratchet effects $[24,25]$ or current switching by laser fields $[35,36,47,48]$.

\section{Acknowledgements}

The authors would like to thank Sébastien Camalet, Gert-Ludwig Ingold, Heiko B. Weber, and Jan Würfel for useful discussions. This work has been supported by the Volkswagen-Stiftung under Grant No. I/77217 and the DFG Sonderforschungsbereich 486, project A10.

\section{References}

1. M.A. Reed, C. Zhou, C.J. Muller, T.P. Burgin, J.M. Tour: Conductance of a molecular junction, Science, 278, 252 (1997)

2. X.D. Cui, A. Primak, X. Zarate, J. Tomfohr, O.F. Sankey, A.L. Moore, T.A. Moore, D. Gust, G. Harris, S.M. Lindsay: Reproducible measurement of singlemolecule conductivity, Science, 294, 571 (2001)

3. J. Reichert, R. Ochs, D. Beckmann, H.B. Weber, M. Mayor, H. von Löhneysen: Driving current through single organic molecules, Phys. Rev. Lett., 88, 176804 (2002)

4. H.B. Weber, J. Reichert, F. Weigend, R. Ochs, D. Beckmann, M. Mayor, R. Ahlrichs, H. von Löhneysen: Electronic transport through single conjugated molecules, Chem. Phys., 281, 113 (2002)

5. P. Hänggi, M. Ratner, and S. Yaliraki, Processes in Molecular Wires, Chem. Phys., 281, 111 (2002).

6. A. Nitzan, M.A. Ratner: Electron transport in molecular wire junctions, Science, 300, 1384 (2003)

7. J.R. Heath, M.A. Ratner: Molecular electronics, Phys. Today, 56(5), 43 (2003)

8. R.M. Metzger: Unimolecular electrical rectifiers, Chem. Rev., 103, 3803 (2003)

9. M. Di Ventra, N.D. Lang: Transport in nanoscale conductors from first principles, Phys. Rev. B, 65, 045402 (2002)

10. J. Heurich, J.C. Cuevas, W. Wenzel, G. Schön: Electrical transport through single-molecule junctions: From molecular orbitals to conduction channels, Phys. Rev. Lett., 88, 256803 (2002)

11. T. Seideman: Current-driven dynamics in molecular-scale devices, J. Phys. C 15, R521 (2003) 
12. T. Seideman, H. Guo: Quantum transport and current-triggered dynamics in molecular tunnel junctions, J. Theo. Comp. Chem., 2, 439 (2003)

13. V. Mujica, M. Kemp, M.A. Ratner: Electron conduction in molecular wires. I. A scattering formalism, J. Chem. Phys., 101, 6849 (1994)

14. D. Segal, A. Nitzan, W.B. Davis, M.R. Wasielewski, M.A. Ratner: Electron transfer rates in bridged molecular systems 2: A steady-state analysis of coherent tunneling and thermal relaxation, J. Phys. Chem., 104, 3817 (2000)

15. D. Boese, H. Schoeller: Influence of nanomechanical properties on singleelectron tunneling: A vibrating single-electron transistor, Europhys. Lett., 54, $668(2001)$

16. E.G. Petrov, P. Hänggi: Nonlinear electron current through a short molecular wire, Phys. Rev. Lett., 86, 2862 (2001)

17. A. Nitzan: Electron transmission through molecules and molecular interfaces, Annu. Rev. Phys. Chem., 52, 681 (2001)

18. G. Cuniberti, G. Fagas, K. Richter: Fingerprints of mesoscopic leads in the conductance of a molecular wire, Chem. Phys., 281, 465 (2002)

19. M.H. Hettler, W. Wenzel, M.R. Wegewijs, H. Schoeller: Current collapse in tunneling transport through benzene, Phys. Rev. Lett., 90, 076805 (2003)

20. S. Kohler, J. Lehmann, P. Hänggi: Driven transport on the nanoscale, Phys. Rep., 406, 379 (2005)

21. C.A. Stafford, N.S. Wingreen: Resonant photon-assisted tunneling through a double quantum dot: An electron pump from spatial rabi oscillations, Phys. Rev. Lett., 76, 1916 (1996)

22. T. Fujisawa, S. Tarucha: Photon assisted tunnelling in single and coupled quantum dot systems, Superlatt. Microstruct., 21, 247 (1997)

23. T.H. Oosterkamp, T. Fujisawa, W.G. van der Wiel, K. Ishibashi, R.V. Hijman, S. Tarucha, L.P. Kouwenhoven: Microwave spectroscopy of a quantum-dot molecule, Nature, 395, 873 (1998)

24. J. Lehmann, S. Kohler, P. Hänggi, A. Nitzan: Molecular wires acting as coherent quantum ratchets, Phys. Rev. Lett., 88, 228305 (2002)

25. J. Lehmann, S. Kohler, P. Hänggi, A. Nitzan: Rectification of laser-induced electronic transport through molecules, J. Chem. Phys., 118, 3283 (2003)

26. A. Keller, O. Atabek, M. Ratner, V. Mujica: Laser-assisted conductance of molecular wires, J. Phys., B 35, 4981 (2002)

27. S. Kohler, J. Lehmann, S. Camalet, P. Hänggi: Resonant laser excitation of molecular wires, Israel J. Chem., 42, 135 (2002)

28. S. Kohler, J. Lehmann, M. Strass, P. Hänggi: Molecular wires in electromagnetic fields, Adv. Solid State Phys., 44, 151 (2004)

29. C. Caroli, R. Combescot, P. Nozieres, D. Saint-James: Direct calculation of the tunneling current, J. Phys. C 4, 916 (1971)

30. D.S. Fisher, P.A. Lee: Relation between conductivity and transmission matrix, Phys. Rev. B, 23, 6851 (1981)

31. Y. Meir, N.S. Wingreen: Landauer formula for the current through an interacting electron region, Phys. Rev. Lett., 68, 2512 (1992)

32. Ya. M. Blanter, M. Büttiker: Shot noise in mesoscopic conductors, Phys. Rep., 336, 1 (2000)

33. U. Fano: Ionization yield of radiations. II. The fluctuations of the number of ions, Phys. Rev., 72, 26 (1947)

34. A.P. Jauho, N.S. Wingreen, Y. Meir: Time-dependent transport in interacting and noninteracting resonant-tunneling systems, Phys. Rev. B, 50, 5528 (1994) 
35. S. Camalet, J. Lehmann, S. Kohler, P. Hänggi: Current noise in ac-driven nanoscale conductors, Phys. Rev. Lett., 90, 210602 (2003)

36. S. Camalet, S. Kohler, P. Hänggi: Shot-noise control in ac-driven nanoscale conductors, Phys. Rev. B, 70, 155326 (2004)

37. S. Datta, M.P. Anantram: Steady-state transport in mesoscopic systems illuminated by alternating fields, Phys. Rev. B, 45, 13761 (1992)

38. S. Datta: Electronic Transport in Mesoscopic Systems (Cambridge University Press, Cambridge, 1995)

39. M. Wagner: Probing Pauli blocking factors in quantum pumps with broken time-reversal symmetry, Phys. Rev. Lett., 85, 174 (2000)

40. H. Sambe: Steady states and quasienergies of a quantum-mechanical system in an oscillating field, Phys. Rev. A, 7, 2203 (1973)

41. M. Grifoni, P. Hänggi: Driven quantum tunneling, Phys. Rep., 304, 229 (1998)

42. A. Buchleitner, D. Delande, J. Zakrzewski: Non-dispersive wave packets in periodically driven quantum systems, Phys. Rep., 368, 409 (2002)

43. J.H. Shirley: Solution of the Schrödinger equation with a Hamiltonian periodic in time, Phys. Rev., 138, B979 (1965)

44. P. Jung, P. Hänggi: Resonantly driven Brownian motion: Basic concepts and exact results, Phys. Rev. A, 41, 2977 (1990)

45. J. Lehmann, S. Kohler, V. May, P. Hänggi: Vibational effects in laser-driven molecular wires, J. Chem. Phys., 121, 2278 (2004)

46. M.A. Ratner: Bridge-assisted electron transfer: Effective electronic coupling, J. Phys. Chem., 94, 4877 (1990)

47. J. Lehmann, S. Camalet, S. Kohler, P. Hänggi: Laser controlled molecular switches and transistors, Chem. Phys. Lett., 368, 282 (2003)

48. S. Kohler, S. Camalet, M. Strass, J. Lehmann, G.L. Ingold, P. Hänggi: Charge transport through a molecule driven by a high-frequency field, Chem. Phys., 296, $243(2004)$ 
